\title{
Metanálise da relação entre espessura de toicinho e variáveis corporais e reprodutivas de porcas gestantes e lactantes
}

\author{
Meta-analysis of relation among backfat thickness, body and reproductive variables of gestating and \\ lactating sows
}

\author{
Carlos Augusto Rigon Rossi ${ }^{\mathrm{I}}$ Paulo Alberto Lovatto ${ }^{\mathrm{II}}$ Volnei Antônio Weschenfelder ${ }^{\mathrm{I}}$ \\ Cheila Roberta Lehnen"II
}

\section{RESUMO}

Um estudo de metanálise avaliou a relação entre a espessura de toicinho e as variáveis de condição corporal de porcas gestantes e lactantes. A base de dados contemplou 14 artigos publicados de 2000 a 2006 em revistas indexadas. A metanálise foi realizada através de análises gráfica, de correlação e de variância. A correlação da espessura de toicinho (ET) com o peso vivo foi de 0,16 ( $P<0,01)$, com a massa protéica de 0,48 $(P<0,01)$ e com a concentração de leptina de $0,88(P<0,01)$. A correlação da variação da espessura de toicinho na lactação (ETl) com o peso vivo foi de $-0,21$ $(P<0,01)$, com variação do peso vivo na lactação de 0,34 $(P<0,01)$ e com variação da massa lipídica na lactação de $0,70(P<0,01)$. A correlação entre a ET e o número de leitões nascidos vivos foi de 0,46 $(P<0,01)$, e entre a ETl e o PV dos leitões aos sete dias foi de 0,95 $(P<0,01)$. A ET foi influenciada pelo peso vivo e pela massa protéica na gestação, enquanto a ETl é influenciada pela variação do peso vivo e pela massa lipídica na lactação. As concentrações de leptina ao parto estão correlacionadas positivamente com a ET. A ET é influenciada pelo número total de leitões nascidos vivos e pelo peso vivo dos leitões ao nascimento, enquanto a ETl é influenciada pelo peso vivo dos leitões aos sete dias e pelo ganho de peso vivo da leitegada. Há relação significativa entre espessura de toicinho e variáveis de condição corporal de porcas gestantes e lactantes.

Palavras-chave: leitões, leptina, massa lipídica, massa protéica, peso vivo.

\section{ABSTRACT}

A meta-analysis was carried out to evaluate the association between backfat thickness and sow body condition in gestation and lactation. The database assembled 14 publications from 2000 to 2006. The meta-analysis was accomplished by graphical analysis, correlation, and analysis of variance. The correlation between backfat thickness (BT) and body weight was $0.16(P<0.01)$, with protein mass was $0.48(P<0.01)$ and leptin concentration was $0.88(P<0.01)$. The correlation between the backfat variation during and in lactation (VBTl) and body weight was $-0.21(P<0.01)$, with body weight variation in lactation was $0.34(P<0.01)$ and with fat mass variation in lactation was $0.70(P<0.01)$. The correlation between $B T$ and born alive litter size was 0.46 $(P<0.01)$, between VBTl and piglets body weight at seven days of age was $0.95(P<0.01)$. In the gestation, the $B D$ was influenced by the body weight and protein mass. However, in lactation the VBTl was influenced by the body weight variation and fat mass. The leptin concentration at farrowing was positively correlated with backfat depth. The BT was influenced by born alive litter size and piglets birth weight. The VBTl was influenced by piglets weight at seven days old and litter weight gain. In conclusion, there is a significant relation between backfat thickness and body variables of the sows in gestation and lactation.

Key words: fat mass, leptin, piglets, protein mass, weight.

\section{INTRODUÇÃO}

O sucesso da suinocultura moderna está relacionado, em grande parte, ao desempenho reprodutivo das porcas (PENZ JR \& EBERT, 2001). A nutrição e a alimentação são os aspectos que mais afetam o desempenho reprodutivo e os que mais contribuem no custo de produção, representando cerca de $12 \%$ do total (LUDKE et al., 2006). As necessidades nutricionais das porcas variam com a idade, o peso

IPrograma de Pós-graduação em Zootecnia, Departamento de Zootecnia, Universidade Federal de Santa Maria (UFSM), 97105900, Santa Maria, RS, Brasil. E-mail: carlos.rossi@hotmail.com. Autor para correspondência.

IDepartamento de Zootecnia, UFSM, Santa Maria, RS, Brasil.

IIICurso de Zootecnia, UFSM, Santa Maria, RS, Brasil. 
vivo e fase reprodutiva. As exigências nutricionais durante a gestação são inferiores as da lactação e dependem da ordem de parto, do estado nutricional, do período da gestação, da estação do ano e da linhagem da porca (FERREIRA et al., 2006).

Na prática, exigências nutricionais das porcas em gestação e lactação são determinadas por um escore que, fundamentado na descrição visual, quantifica a condição corporal num índice de 1 a 5 (YOUNG \& AHERNE, 2005a). O uso do escore corporal permite classificar as porcas em grupos homogêneos adequando-os às dietas (DOURMAD et al., 2001). No entanto, a avaliação visual das porcas é um critério subjetivo, podendo gerar situações de sub ou super alimentação (YOUNG et al., 2004).

A avaliação da condição corporal de porcas gestantes e lactantes pode ser baseada também em variáveis objetivas como a espessura de toicinho (ET), a ordem de parto e o peso vivo (AHERNE, 2004) e a concentração de leptina (RENSIS et al., 2005). A leptina é uma proteína de $16 \mathrm{kDa}$ secretada pelos adipócitos e envolvida na regulação do apetite, no metabolismo energético e na reprodução. As variáveis objetivas podem ser usadas para adequar os requerimentos nutricionais e reduzir o custo anual de alimentação (YOUNG \&AHERNE, 2005a).

Nas condições tecnológicas atuais, o uso de variáveis subjetivas para a definição de programas alimentares e nutricionais de porcas pode não ser suficiente. Mas as informações disponíveis sobre o ajuste nutricional através de variáveis objetivas são incipientes e pouco conclusivas, quando analisadas de forma isolada. Como as pesquisas em ciência animal dependem de métodos estatísticos, as relações quantitativas podem ser mais bem exploradas através da metanálise. Este método permite combinar os resultados de estudos realizados de forma independente (extraídos de trabalhos publicados) e sintetizar suas conclusões, ou mesmo obter uma nova conclusão (LUIZ, 2002). Este trabalho tem o objetivo de avaliar de forma sistêmica, através da metanálise, a relação entre a espessura de toicinho e as variáveis de condição corporal de porcas gestantes e lactantes.

\section{MATERIAL E MÉTODOS}

O trabalho foi realizado no Setor de Suinocultura do Departamento de Zootecnia da Universidade Federal de Santa Maria e no Grupo de Modelagem Animal (Grupos de Pesquisa CNPq). Foram utilizados 14 artigos distribuídos nos continentes europeu e americano, publicados de 2000 a 2006 em revistas indexadas internacionais (PRUNIER et al., 2001,
SINCLAIR et al., 2001; COOPER et al., 2001a, CLOWES et al., 2003; MAES et al., 2004; RAMANAU et al., 2004; VAN DER PEET-SCHWERING et al., 2004; YOUNG et al., 2004; RENSIS et al., 2005; YOUNG et al., 2005b; LANDBLOM et al., 2006) e nacionais (GUEDES \& NOGUEIRA, 2000; HASHIMOTO et al., 2004; LIMA et al., 2006). As informações relevantes das seções de material e métodos e resultados de cada artigo foram inseridas numa base de dados elaborada em planilha Excel. A metodologia utilizada para definição das variáveis dependentes e independentes e codagem dos dados seguiu proposições descritas na literatura (LOVATTO, 2003; SAUVANT et al., 2005). A codagem dos dados seguiu critérios qualitativos de agrupamento. De forma objetiva, a codagem é um recurso utilizado em metanálise para associar grupos homogêneos em determinados critérios, o que permite incluí-los nos modelos analíticos como fonte de variação. As principais codagens foram a espessura de toicinho (ETco), os tratamentos (TRATco), peso vivo (PVco) e ordem de parto (OPco). A base de dados utilizada na metanálise totalizou 560 tratamentos de 24 animais cada (7 a 324 animais), 12.635 porcas gestantes e lactantes, espessura de toicinho média de 17,44mm (4,5 a 28,1mm), avaliada no final da gestação, período de lactação de 21 dias e peso médio de 235kg (149 a $334 \mathrm{~kg})$. A metanálise foi realizada segundo SAUVANT et al. (2005) e seguiu três análises seqüenciais: gráfica, de correlação e de variância. Na análise gráfica, foi estudada a coerência biológica dos dados, propriedade em que o pesquisador pode avaliar, pelo seu conhecimento acumulado, se as informações sistêmicas da base de dados têm coerência com as respostas biológicas esperadas. Na análise de correlação, foram identificadas as variáveis correlacionadas na base. Na análise de variância (ANOVA), foram incluídos no modelo os fatores que variaram em função da significância das correlações. As equações de regressão foram obtidas através da covariância contínua nos modelos de ANOVA. As análises descritivas e estatísticas foram realizadas através do programa MINITAB (MCKENZIE \& GOLDMAN, 1999).

\section{RESULTADOS E DISCUSSÃO}

Os coeficientes de correlação entre ET, variação na espessura de toicinho na lactação (ETl) e variáveis corporais de porcas gestantes e lactantes são apresentados na tabela 1. Entre as variáveis peso vivo (PV), massa protéica (MP) e massa lipídica (ML), a MP foi a que apresentou maior correlação com a ET $(0,48 ; \mathrm{P}<0,01)$. As variáveis com maior correlação com 
Tabela 1 - Coeficientes de correlação da espessura de toicinho com variáveis corporais de porcas e leitões.

\begin{tabular}{|c|c|c|c|c|c|}
\hline \multirow{2}{*}{ Resposta } & \multicolumn{5}{|c|}{ Variáveis } \\
\hline & $\begin{array}{l}\text { Consumo ração gestação, } \\
\qquad \mathrm{kg} \mathrm{dia}^{-1}\end{array}$ & $\begin{array}{l}\text { Consumo ração } \\
\text { lactação, kg dia }{ }^{-1}\end{array}$ & $\begin{array}{l}\text { Consumo proteína, } \\
\text { g dia }^{-1}\end{array}$ & $\begin{array}{l}\text { Consumo total, } \\
\text { kg }\end{array}$ & $\begin{array}{l}\mathrm{PV}, \\
\mathrm{kg}\end{array}$ \\
\hline ET & $-0,125$ & $-0,022$ & 0,386 & $-0,325$ & 0,165 \\
\hline $\mathrm{P}$ & 0,407 & 0,765 & 0,305 & 0,080 & 0,001 \\
\hline $\mathrm{ETl}$ & 0,488 & $-0,205$ & $-0,623$ & 0,375 & $-0,212$ \\
\hline $\mathrm{P}$ & 0,016 & 0,008 & 0,073 & 0,168 & 0,002 \\
\hline Porcas & PV, variação lactação, kg & Massa protéica, kg & Massa lipídica, kg & MLl, kg & Leptina ao parto, $\mathrm{ng} \mathrm{ml}^{-1}$ \\
\hline ET & 0,126 & 0,480 & 0,235 & $-0,114$ & 0,879 \\
\hline $\mathrm{P}$ & 0,059 & 0,000 & 0,047 & 0,508 & 0,000 \\
\hline ETl & 0,344 & $-0,274$ & 0,156 & 0,705 & 0,333 \\
\hline $\mathrm{P}$ & 0,000 & 0,106 & 0,363 & 0,000 & 0,381 \\
\hline Porcas & $\mathrm{LNV}, \mathrm{n}^{\circ}$ & $\mathrm{LN}, \mathrm{n}^{\circ}$ & $\mathrm{LD}, \mathrm{n}^{\circ}$ & PVLN, kg & PVL7, kg \\
\hline ET & 0,465 & $-0,147$ & 0,011 & 0,176 & 0,817 \\
\hline $\mathrm{P}$ & 0,008 & 0,065 & 0,882 & 0,026 & 0,047 \\
\hline ETl & 0,498 & 0,154 & $-0,057$ & 0,460 & 0,954 \\
\hline $\mathrm{P}$ & 0,315 & 0,692 & 0,430 & 0,359 & 0,003 \\
\hline Porcas & PVL14, kg & PVLD, kg & GPV leitão, kg & GPV & eitegada, kg \\
\hline ET & 0,614 & $-0,151$ & $-0,156$ & & $-0,269$ \\
\hline $\mathrm{P}$ & 0,079 & 0,044 & 0,060 & & 0,227 \\
\hline ETl & 0,665 & 0,115 & 0,064 & & 0,508 \\
\hline $\mathrm{P}$ & 0,150 & 0,130 & 0,453 & & 0,016 \\
\hline
\end{tabular}

ET-Espessura de toicinho; ETl- Variação da ET na lactação; GPV- Ganho de peso vivo; LD- Leitões desmamados; LNV- Leitões nascidos vivos; LN- Leitões natimortos; MLl- Massa lipídica, variação na lactação; PV- Peso vivo; PVLN- Peso vivo dos leitões ao nascimento; PVL7- Peso vivo dos leitões aos 7 dias após nascimento; PVL14- Peso vivo dos leitões aos 14 dias após nascimento; PVLD- Peso vivo dos leitões ao desmame.

a ETl foram a variação da ML na lactação $(0,70 ; \mathrm{P}<0,01)$ e a variação do $P V$ na lactação $(0,34 ; \mathrm{P}<0,01)$.

O consumo de alimentos, a perda de peso e perda de proteína na lactação são menores em sistemas de produção que monitoram as reservas corporais (YOUNG \& AHERNE, 2005a). A nutrição de porcas gestantes deve maximizar a retenção protéica e garantir uma adequada deposição de gordura. Essa estratégia aumenta a liberação de insulina e reduz os níveis de glucagon, aumentando o consumo voluntário de alimentos na lactação (KIM \& EASTER, 2006). A dieta deve garantir um mínimo de tecido magro contra os efeitos do catabolismo lactacional, que reduz cerca de $11 \%$ de massa protéica (CLOWES et al., 2003). As correlações mostram maior influência do PV e MP na gestação e maior influência da variação do PV e ML na lactação.

A concentração de leptina apresentou alta correlação com a ET $(0,88 ; \mathrm{P}<0,01)$. Concentrações elevadas de leptina no soro e no leite, também estão correlacionadas positivamente com a massa lipídica corporal após 4 ou 25 dias de lactação e com a redução do apetite na lactação. A leptina é sintetizada nos adipócitos e sua liberação é inibida por alterações nas reservas de gordura corporal na lactação. Os sinais da leptina são traduzidos pelo sistema neuroendócrino na regulação do apetite, na liberação do hormônio liberador de gonadotrofinas (GnRH) e na secreção do hormônio luteinizante (LH) (PRUNIER et al., 2001). Os resultados evidenciados nesta metanálise mostram que concentrações de leptina na lactação podem ser utilizadas como variáveis em programas nutricionais de porcas, visando a regular o apetite e a melhorar o desempenho reprodutivo desses animais.

A correlação entre a ET e o número de leitões nascidos vivos foi de $0,46(\mathrm{P}<0,01)$. Uma ET entre $14 \mathrm{e}$ $15,5 \mathrm{~mm}$ no final da gestação pode reduzir o número de leitões nascidos vivos (MAES et al., 2004). No entanto, uma ET superior a 20mm ao parto predispõe distocia, agalactia, mastite e metrite (WELDON et al., 1994). Assim, a correlação positiva entre ET e nascidos vivos demonstra a importância de monitorar a condição 
corporal da porca. A ordem de parto também deve ser levada em consideração, pois o número total de leitões nascidos e o número de leitões nascidos vivos são menores em primíparas (COOPER et al., 2001b).

O PV dos leitões apresentou correlação com ET de $0,18(\mathrm{P}=0,02)$, correlação com ET aos sete dias de $0,81(\mathrm{P}=0,05)$, correlação com ET aos 14 dias de 0,61 $(\mathrm{P}=0,08)$ e correlação com ET ao desmame de $-0,15$ $(\mathrm{P}=0,04)$. O maior consumo de ração e/ou energia pelas porcas no final do período de gestação aumenta o peso dos leitões ao nascimento (CROMWELL et al., 1989). Cada quilograma de ganho de peso total da porca na gestação resulta em $50 \mathrm{~g}$ de peso adicional da leitegada ao nascimento (COOPER et al., 2001b). Leitões mais pesados ao nascimento podem ganhar até $170 \mathrm{~g}$ a mais no peso ao desmame (CROMWELL et al., 1989).

A correlação da ETl com o PV aos sete dias após o nascimento foi de $0,95(\mathrm{P}<0,01)$. O número de leitões desmamados por porca e a redução da ETl estão associados à exigência mais elevada para a lactogênese. A ETl pode ser reduzida em 15\%, diminuindo o tempo de lactação e aumentando o consumo voluntário de alimentos (MAES et al., 2004).

Os resultados da análise de variância da ET utilizando PV, MP e ML são apresentados na tabela 2. Dos fatores utilizados no modelo, as codagens de tratamentos e a ordem de parto apresentaram os melhores coeficientes de determinação. A ET varia em função da MP e da ML, quando a ordem de parto é incluída no modelo (ET = 14,54 - 0,68 MP + 0,59 ML). Para cada quilograma de massa protéica há uma redução de $0,68 \mathrm{~mm}$ da espessura de toicinho, e para cada quilograma de massa lipídica há um aumento de $0,59 \mathrm{~mm}$ na espessura de toicinho. A ordem de parto é importante, pois as exigências de energia para ganhos de peso e ET de pluríparas são maiores do que para as primíparas (DOURMAD et al., 1998). As primíparas exigem mais proteína para ganho de peso, em comparação ao ganho de massa lipídica de pluríparas (TESS et al., 1984). O ganho de peso estimado na gestação, para primíparas, representa 25\% de tecido lipídico e 75\% de tecido magro (PETTIGREW \& YANG, 1997). Dessa forma, a nutrição das porcas de primeiro e segundo partos deve ser diferenciada do restante do plantel.

Os resultados da ETl em função do PV e da variação do PV na lactação são apresentados na tabela 3. Os coeficientes de determinação para a variação do PV na lactação foram de 64 e $65 \%$ para as codagens ET e PV, respectivamente. As porcas devem consumir grandes quantidades de alimento na gestação para que, no início da lactação, consigam adaptar-se à demanda da produção de leite (REVELL et al., 1998). Entretanto, o maior consumo alimentar na gestação diminui o consumo voluntário de alimentos na lactação, o qual é associado ao acúmulo de gordura corporal (WELDON, 1994). Em função do baixo consumo voluntário de alimentos, as porcas perdem peso, aumentando o intervalo desmama-estro, reduzindo a taxa de prenhez e diminuindo o tamanho de leitegada subseqüente (PETTIGREW \& YANG, 1997).

A ETl em função do peso vivo e da variação do peso vivo na lactação apresentou o maior coeficiente de determinação (67\%), quando os tratamentos foram incluídos no modelo. A ETl em função das covariáveis PV e da variação do PV na lactação depende dos tratamentos incluídos no modelo (ETl = 2,87 - 0,007 PV + 0,01 PV, variação PV na lactação). O peso vivo durante a lactação varia em função da mobilização de gordura e proteína corporais (PETTIGREW \& YANG, 1997). A mobilização de gordura varia de 68 a 81\% (REVELL et al., 1998). A ETl e a variação do peso vivo na lactação

Tabela 2 - Equações para espessura de toicinho na gestação (ET) obtidas por análise de covariância contínua utilizando covariáveis corporais.

\begin{tabular}{lllllll}
\hline Resposta & Fatores & Covariáveis & N & dpr & R2, \% & Equação \\
\hline ET, mm & ETco & PV, kg & 409 & 3,08 & 39,1 & ET=6,76 + 0,05 PV \\
ET, mm & TRATco & PV, kg & 409 & 3,2 & 40,5 & ET= 6,008 + 0,05 PV \\
ET, mm & PVco & PV; ML, kg & 26 & 3,34 & 43,8 & ET= 11,92 + 0,05 PV - 0,12 ML \\
ET, mm & PVco & PV; MP, kg & 26 & 3,23 & 47,4 & ET= 8,71 - 0,008 PV + 0,28 MP \\
ET, mm & PVco & MP; ML, kg & 36 & 2,88 & 50,5 & ET= 9,28 + 0,22 MP - 0,012 MP \\
ET, mm & TRATco & PV; MP, kg & 52 & 1,36 & 94,6 & ET= - 16,63 + 0,07 PV + 0,51 MP \\
ET, mm & TRATco & MP; ML, kg & 72 & 0,62 & 98,7 & ET= 7,431 - 0,3 MP + 0,39 ML \\
ET, mm & TRATco & PV; ML, kg & 52 & 0,64 & 98,8 & ET= - 4,09 + 0,03 PV + 0,26 ML \\
ET, mm & OPco & MP, ML, kg & 18 & 0,21 & 99,6 & ET= 14,54 - 0,68 MP + 0,59 ML \\
\hline
\end{tabular}

ETco- Codagem espessura de toicinho; ET- espessura de toicinho; ML- Massa lipídica; MP- Massa protéica; OPco- Codagem ordem de parto; PVco- Codagem peso vivo; PV- Peso vivo; TRATco- Codagem tratamentos. 
Tabela 3 - Equações para espessura de toicinho na lactação (ETl) obtidas por análise de covariância contínua utilizando covariáveis corporais.

\begin{tabular}{|c|c|c|c|c|c|c|}
\hline Resposta & Fatores & Covariáveis & $\mathrm{N}$ & dpr & $\mathrm{R} 2, \%$ & Equação \\
\hline ETl & ETco & $\mathrm{PV}, \mathrm{kg}$ & 217 & 1,19 & 63,6 & $\mathrm{ETl}=3,74-0,008 \mathrm{PV}$ \\
\hline ETl & PVco & PV, kg & 217 & 1,18 & 64,1 & $\mathrm{ETl}=3,87-0,0089 \mathrm{PV}$ \\
\hline ETl & ETco & $\begin{array}{l}\text { PV, variação PV na } \\
\text { lactação, kg }\end{array}$ & 217 & 1,17 & 65,1 & $\begin{array}{l}\mathrm{ETl}=2,65-0,005 \text { PV + 0,01 PV, variação PV } \\
\text { na lactação }\end{array}$ \\
\hline ETl & PVco & $\begin{array}{l}\text { PV, variação PV na } \\
\text { lactação, kg }\end{array}$ & 217 & 1,16 & 65,5 & $\begin{array}{l}\mathrm{ETl}=2,79-0,005 \mathrm{PV}+0,016 \mathrm{PV} \text {, variação } \\
\mathrm{PV} \text { na lactação }\end{array}$ \\
\hline $\mathrm{ETl}$ & TRATco & $\mathrm{PV}, \mathrm{kg}$ & 217 & 1,21 & 66,1 & $\mathrm{ETl}=3,95-0,01 \mathrm{PV}$ \\
\hline ETl & TRATco & $\begin{array}{l}\text { PV, variação PV na } \\
\text { lactação, kg }\end{array}$ & 217 & 1,20 & 67,3 & $\begin{array}{l}\mathrm{ETl}=2,87-0,007 \mathrm{PV}+0,01 \mathrm{PV} \text {, variação PV } \\
\text { na lactação }\end{array}$ \\
\hline
\end{tabular}

ET-espessura de toicinho; ETco- Codagem espessura de toicinho; ETl- Variação da ET na lactação; MS- Massa seca; OPco- Codagem ordem de parto; PVco- Codagem peso vivo; PV- Peso vivo; TRATco- Codagem tratamentos.

ocorrem em função do consumo voluntário de alimentos. É por isso que porcas superalimentadas na gestação depositam mais gordura corporal até o parto, reduzindo o consumo na lactação (REVELL et al., 1998).

Os resultados da análise de variância entre a ET, a ETl e o desempenho dos leitões são apresentados na tabela 4. Os melhores resultados foram obtidos utilizando-se a codagem da ET como fator de análise. O maior coeficiente de determinação para a ET foi observado com o número de leitões nascidos vivos (99,8\%). A ET é influenciada pelo número de leitões nascidos vivos (ET $=13,32+0,39$ total nascidos), pois para cada leitão nascido a mais corresponde ao aumento de 0,39mm na ET. As exigências nutricionais das porcas são para mantença, ganho de peso, crescimento fetal e produção de leite (MAROTTA \& LAGRECA, 2003). O acúmulo de massa lipídica, na gestação, depende do nível alimentar (CLOSE et al., 1985). As exigências energéticas do útero e seu conteúdo estão diretamente relacionados com o tamanho da leitegada, pois cada quilograma de peso vivo do leitão ao nascimento corresponde a 1,3Mcal de EM, com uma eficiência de utilização de 40 a 50\% (NOBLET \& ETIENNE, 1987).

Para a ETl, o melhor resultado foi entre ETco e ganho de $\mathrm{PV}$ da leitegada $(\mathrm{R}=88 \%)$. A ETl é influenciada pelo ganho de PV da leitegada (ETl = 8,05 - 0,21 ganho PV leitegada). O aumento no consumo voluntário de alimentos na lactação reduz as perdas de ET e PV das porcas e aumenta o ganho de peso da leitegada (EISSEN et al., 2003). A relação entre ET, ETl e desempenho dos leitões sugere que as exigências energéticas de porcas na gestação são fundamentais tanto para recuperar o escore corporal da porca, como para o desempenho futuro dos leitões. Nesse contexto, as variáveis objetivas podem ser usadas para monitorar a condição corporal de porcas gestantes e lactantes, com o intuito de melhorar o desempenho produtivo e reprodutivo.

\section{CONCLUSÕES}

A espessura de toicinho na gestação é influenciada pelo peso vivo e pela massa protéica das

Tabela 4 - Equações para espessura de toicinho (ET) e espessura de toicinho na lactação (ETl) obtidas por análise de covariância contínua utilizando covariáveis corporais e o desempenho de leitões.

\begin{tabular}{lllllll}
\hline Variável & Fatores & \multicolumn{1}{c}{ Covariáveis } & $\mathrm{N}$ & $\mathrm{dpr}$ & $\mathrm{R} 2, \%$ & \multicolumn{1}{c}{ Equação } \\
\hline ET & TRATco & PV leitão desmame, kg & 179 & 3,43 & 21,4 & ET= 18,78 - 0,41 PV \\
ET & ETco & PV leitões nascimento & 160 & 3,48 & 27 & ET= 13,90 + 3,12 PV leitões \\
ET & TRATco & PV leitão nascimento, kg & 160 & 3,54 & 27,1 & ET= 13,7 + 3,12 PV \\
ET & ETco & PV leitão 7 dias & 6 & 0,31 & 70,6 & ET= 8,23 + 3,36 PV 7 dias \\
ETl & ETco & Ganho PV leitegada, kg & 22 & 0,74 & 88,2 & ETl= 8,05 - 0,21 Ganho PV leitegada \\
ETl & ETco & PV leitão 7dias & 6 & 0,2 & 90,9 & ETl= -8,37 + 4,53 PV leitão \\
ET & ETco & № leitões, nascidos vivos & 31 & 0,49 & 99,8 & ET= 13,32 + 0,39 total nascidos \\
\hline
\end{tabular}

ET- espessura de toicinho; ETco- Codagem espessura de toicinho; ETl- Variação da ET na lactação; TRATco- Codagem de tratamentos; PVPeso vivo.

Ciência Rural, v.38, n.1, jan-fev, 2008. 
porcas. A espessura de toicinho na lactação é influenciada pelo peso vivo e pela massa lipídica.

A espessura de toicinho ao parto é influenciada pelo número total de leitões nascidos vivos e pelo peso vivo dos leitões ao nascimento. A variação da espessura de toicinho na lactação é influenciada pelo peso vivo dos leitões aos sete dias e pelo ganho de peso da leitegada.

A espessura de toicinho é correlacionada positivamente com a concentração de leptina.

\section{AGRADECIMENTOS}

À Coordenação de Aperfeiçoamento de Pessoal de Nível Superior (CAPES), pela concessão de bolsa ao mestrando do Programa de Pós-graduação em Zootecnia da Universidade Federal de Santa Maria (UFSM) Volnei Antônio Weschenfelder. À UFSM, pela concessão de bolsa do Fundo Institucional de Pesquisa (FIPE) a Cheila Roberta Lehnen. Ao Grupo de Modelagem Animal (Grupos de Pesquisa CNPq), pela infraestrutura para a realização do trabalho.

\section{REFERÊNCIAS}

AHERNE, F. Sow backfat and weight watchers. Capturado em 22 mar. 2004. Online. Disponível na internet http:// nationalhogfarmer.com/mag/farming_sow_backfat_weight/ index.html

CLOSE, W.H. et al. Studies on the energy metabolism of the pregnant sow. British Journal of Nutrition, v.53, p.267279, 1985.

CLOWES, E.J. et al. Selective protein loss in lactating sows is associated with reduced litter growth and ovarian function. Journal of Animal Science, v.81, p.753-764, 2003.

COOPER, D.R. et al. Effect of nutrient intake in lactation on sow performance: determining the threonine requirement of the high-producing lactating sow. Journal of Animal Science, v.79, p.2378-2387, 2001a.

COOPER, D.R. et al. Effect of energy and lysine intake in gestation on sow performance. Journal of Animal Science, v.79, p.2367-2377, 2001b.

CROMWELL, G.L. et al. Effects of additional feed during late gestation on reproductive performance of sows: a cooperative study. Journal of Animal Science, v.67, p.3-14, 1989.

DOURMAD, J.Y. et al. Mesurer l'épaisseur de lard dorsal des truies pour définir leurs programmes alimentaires. INRA Productions Animales, v.14, p.41-50, 2001.

DOURMAD, J.Y. et al. Effect of protein and lysine supply on performance, nitrogen balance, and body composition changes of sows during lactation. Journal of Animal Science, v.76, p.542-550, 1998.

EISSEN, J.J. et al. The importance of a high feed intake during lactation of primiparous sows nursing large litters. Journal of Animal Science, v.81, p.594-603, 2003.

FERREIRA, A.S. et al. Níveis de proteína bruta na ração para porcas pluríparas em gestação. Revista Brasileira de Zootecnia, v.35, p.761-767, 2006.
GUEDES, R.M.C.; NOGUEIRA, R.H.G. Relationship among body condition at parturition, decrease of backfat thickness and weight during the lactation and the interval from weaning to oestrus of sows. Brazilian Journal of Veterinary Research and Animal Science, São Paulo, v.37, 2000. Capturado em 13 de dez. 2006. Online. Disponível na internet: http://www.scielo.br/scielo.php?script=sci_arttext\&pid

HASHIMOTO, F.A. et al. Níveis de proteína bruta na ração de gestação para porcas de segundo e terceiro ciclos reprodutivos. Revista Brasileira de Zootecnia, v.33, p.365-374, 2004.

KIM, S.W.; EASTER, R.A. Establishing nutrient requirements for the lactating sow: A summary of recent Illinois research. Capturado em 30 abr. 2006. Online. Disponível na internet http://www.traill.uiuc.edu/uploads/ porknet/papers/NutrientRev\%2Epdf

LANDBLOM, D.G. et al. An evaluation of the replacement value of field peas for soybean meal in sow lactation diets. Capturado em 25 abr. 2006. Online. Disponível na internet http://www.ag.ndsu.nodak.edu/dickinso/research/2000/ swine00b.htm

LIMA, K.R.S. et al. Desempenho de porcas submetidas durante a gestação do primeiro ao terceiro parto a dietas com diferentes níveis de proteína bruta. Revista Brasileira de Zootecnia, v.35, n.5, p.1999-2006, 2006.

LOVATTO, P.A. Princípios de modelagem e sua aplicação no estudo de cadeias de produção agrícola. In: REUNIÃO ANUAL DA SOCIEDADE BRASILEIRA DE ZOOTECNIA, 2003, Santa Maria. Anais... Santa Maria: SBZ, 2003. p.1-22.

LUDKE, J.V. et al. Alimentação das fêmeas suínas segundo sua condição corporal. 2000. Capturado em 12 set. 2006. Online. Disponível na internet http://www.cnpsa.embrapa.br.

LUIZ, A.J.B. Meta-análise: definição, aplicações e sinergia com dados espaciais. Cadernos de Ciência \& Tecnologia, v.19, n.3, p.407-428, 2002.

MAES, D.G.D. et al. Back fat measurements in sows from three commercial pig herds: relationship with reproductive efficiency and correlation with visual body condition scores. Livestock Production Science, v.91, p.57-67, 2004.

MAROTTA, E.; LAGRECA, L. Determinación del requerimiento energético de la cerda reproductora mantenida a campo en base al clima y la etología. Analecta Veterinaria, v.23, p.28-35, 2003.

MCKENZIE, J.; GOLDMAN, R.N. The student edition of minitab for windows manual. Belmont: Softcover ed. Addison-Wesley Longman, 1999, V.12, p.592.

NOBLET, J.; ETIENNE, M. Metabolic utilization of energy and maintenance requirements in pregnant sows. Livestock Production Science, v.16, p.243-257, 1987.

PENZ JR, A.M.; EBERT, A.R. Fatores nutricionais que influenciam o peso e a uniformidade dos leitões ao nascimento. In: CONGRESSO BRASILEIRO DE VETERINÁRIOS ESPECIALISTAS EM SUÍNOS, 2001, Porto Alegre. Anais... Porto Alegre: ABRAVES, 2001. p.26-36. 
PETTIGREW, J.E.; YANG, H. Protein nutrition of gestating sows. Journal of Animal Science, v.75, p.2723-2730, 1997.

PRUNIER, A. et al. Influence of feed intake during pregnancy and lactation on fat body reserve mobilisation, plasma leptin and reproductive function of primiparous lactating sows. Reproduction Nutritional Development, v.41, p.333, 2001.

RAMANAU, A. et al. Supplementation of sows with L-Carnitine during pregnancy and lactation improves growth of the piglets during the suckling period through increased milk production. Americam Society for Nutritional Sciences, v.134, p.8692, 2004.

RENSIS, F.D. et al. Relationships between backfat depth and plasma leptin during lactation and sow reproductive performance after weaning. Animal Reproduction Science, v.90, p.95100, 2005.

REVELL, D.K. et al. Body composition at farrowing and nutrition during lactation affect the performance of primiparous sows: I. Voluntary feed intake, weight loss, and plasma metabolites. Journal of Animal Science, v.76, p.1729-1737, 1998.

SAUVANT, D. et al. Les méta-analyses des données expérimentales: applications en nutrition animale. INRA Productions Animales, v.18, p.23-33, 2005.

SINCLAIR, A.G. et al. The influence of gestation feeding strategy on body composition of gilts at farrowing and response to dietary protein in a modified lactation. Journal of Animal Science, v.76, p.2397-2405, 2001.

TESS, M.W. et al. Energy costs of protein and fat deposition in pigs fed ad libitum. Journal of Animal Science, v.58, p.111-122, 1984.

VAN DER PEET-SCHWERING, C.M.C. et al. Effects of additional starch or fat in late-gestating high nonstarch polysaccharide diets on litter performance and glucose tolerance in sows. Journal of Animal Science, v.82, p.2964-2971, 2004.

WELDON, W.C. et al. Postpartum hypophagia in primiparous sows: I. Effects of gestation feeding level on feed intake, feeding behavior, and plasma metabolite concentrations during lactation. Journal of Animal Science, v.72, p.387-394, 1994.

YOUNG, M.; AHERNE, F. Monitoring and maintaining sow condition. Advances in Pork Production, v.16, p.299-313, $2005 a$.

YOUNG, M.G. et al. Comparison of three methods of feeding sows in gestation and the subsequent effects on lactation performance. Journal of Animal Science, v.82, p.30583070, 2004.

YOUNG, M.G. et al. Effect of sow parity and weight at service on target maternal weight and energy for gain in gestation. Journal of Animal Science, v.83, p.255-261, 2005b. 